\title{
Computer Algebra for Analyzing the Vibrations of Nonlinear Structures
}

\author{
Algimantas Čepulkauskas, Regina Kulvietienè, and Genadijus Kulvietis \\ Department of Information Technologies, Vilnius Gediminas Technical University, \\ Saulètekio al. 11, LT-2054, Vilnius, Lithuania \\ acepulka@ktl.mii.lt
}

\begin{abstract}
The aim of this paper is to describe a theoretical background of systematic computer algebra methods for analyzing the free and steady-state periodic vibrations of the nonlinear structures. Many analytical steady-state solution methods are developed but each of them has different capabilities. On the other hand, it is very important to assess the efficiency of analytical methods in terms of the computer algebra system. For this reason, the computer algebra system VIBRAN was used. In this paper, the efficiency of two analytical methods is assessed from the standpoint of the computer algebra system.
\end{abstract}

\section{Introduction}

When a nonlinear structure is subjected to harmonically varying forces, it first passes through a transient state and afterwards it reaches a steady-state or executes chaotic motions. Some steady-states can be non-periodic, but mostly the periodic steady-states are observed. Two related problems of interest are the periodic behavior of structure undergoing harmonic excitation and a free vibration of structure.

Natural vibration of nonlinear systems is of primary concern in studying the resonance phenomena because the backbone curves (the amplitude-frequency relations) and the modes of vibrations, i.e. dynamic characteristics of systems, are determined. Analytical expressions for the backbone curves are very complex and numerical methods are not a convenient way to analyze nonlinear oscillations.

In some cases, such as the one in a range where the internal resonance exists, the corresponding backbone curves have a very complex shape owing to the presence of sharp peaks, looping characteristic and rapidly changing slopes. It is difficult to determine these types of backbone curves by the developed numerical methods [1], [2]. Simulations by means of numerical methods are powerful tools for investigations in mechanics, however, they have serious drawbacks, e.g., finite precision, difficulties in determining transient states and steady-states, the investigation of stability is errorprone and complex.

The analytical steady-state solution by hand requires a lot of routine work, is errorprone and available only for very simple systems. Here the computerized symbolic manipulation systems - so called computer algebra - are indispensable 
tools. Symbolic manipulations provided by computer algebra systems in combination with high-power number crunching abilities of traditional hardware and software really opens a new way to large-scale computations needed in steadystate solutions and the stability analysis [3], [5], [9].

The aim of this paper is to describe a theoretical background of systematic computer algebra methods for analyzing the free and steady-state periodic vibrations of the nonlinear structures. Many analytical steady-state solution methods are developed, but each of them has different capabilities, e.g., small parameter methods give a solution in close form and the harmonic balance method only converts nonlinear differential equations to algebraic. On the other hand, it is very important to assess the efficiency of analytical methods in terms of view of a computer algebra system. For this reason, the computer algebra system VIBRAN 4 was used. The computer algebra system VIBRAN is a FORTRAN preprocessor for analytical computation with polynomials, rational functions, and trigonometric series.

The main VIBRAN distinction from other computer algebra systems is the representation form of analytical expressions. Analytical expressions are stored in matrix form and analytical perturbations are replaced by matrix operations. Analytical operations could be performed by calling VIBRAN procedures directly from the FORTRAN code.

Figure 1 illustrates the VIBRAN procedure fragment to perform Fourier transformation. A special VIBRAN procedure can generate an optimized FORTRAN code from the obtained analytical expressions, that can be directly used in the programs for numerical analysis. In this paper efficiency of two analytical methods is assessed by point of view of computer algebra system VIBRAN.

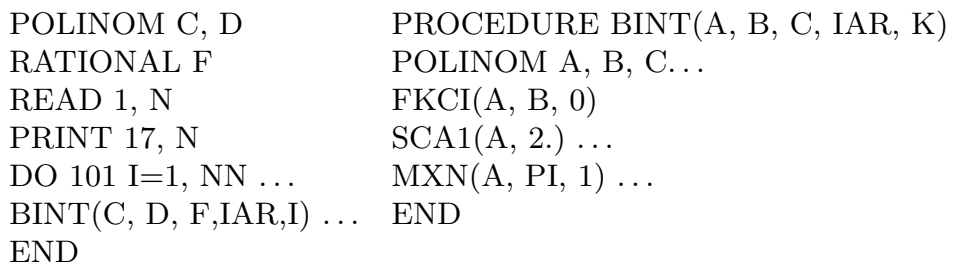

Fig. 1. A fragment of VIBRAN procedures.

\section{Realization of a Small Parameter Method}

The small parameter method (Poincare method) [7] was developed to solve systems of quazi-nonlinear differential equations. Consider the algorithm of the small parameter method realized by the computer algebra system VIBRAN for systems of nonlinear differential equations. For the sake of clarity, an algorithm of the small parameter method is presented for one equation below: 


$$
\ddot{x}+k^{2} x+f(t)=\mu F(t, x, \dot{x}, \mu) .
$$

where $x, \dot{x}, \ddot{x}$ - displacement, velocity and acceleration;

$\mu$ - small parameter;

$f(t)$ - continuous periodical time function with a period $2 \pi$;

$k$ - constant, not integer in nonresonant case and integer in a resonant case;

$F(t, x, \dot{x}, \mu)$ - integer polynomial function and periodical with respect to $t$.

The solution of equation (1) by the small parameter method [7] could be found in the form:

$$
x=x_{0}+\mu x_{1}+\mu^{2} x_{2}+\mu^{3} x_{3}+\ldots,
$$

where $x_{0}$ is the solution of equation (1) without nonlinearities, $x_{i}, i=1,2,3, \ldots$ are unknown functions of $t$ with a period $2 \pi$.

To find these functions, the series (2) is substituted into equation (1) and coefficients with the corresponding $\mu$ power are equalized. With respect to $\mu$ power, linear differential equations are obtained:

$$
\ddot{x}_{i}+k^{2} x_{i}=F_{i}, \quad i=1,2,3, \ldots,
$$

where $F_{i}$ are integer rational functions of $x_{0}, x_{1}, \ldots, x_{i-1} ; \dot{x}_{0}, \dot{x}_{1}, \ldots, \dot{x}_{i-1}$ and continuous periodical function with respect to $t$. The solution of equations (3) in non resonant case could be found in the form:

$$
x_{i}=\frac{a_{i 0}}{2 k^{2}}+\sum_{j=1}^{\infty} \frac{a_{i j} \cos (j t)+b_{i j} \sin (j t)}{k^{2}-j^{2}},
$$

where $a_{i j}$ and $b_{i j}$ are Fourier series coefficients of function $F_{i}$.

In the resonant case, where $k$ is integer and nearly equal or equal to $n$, that means

$$
n^{2}-k^{2}=\varepsilon \mu
$$

where $\varepsilon$ has finite value, the zero power solution must be expressed in the form

$$
\begin{aligned}
& x_{0}=\varphi_{0}(t)+M_{0} \cos (n t)+N_{0} \sin (n t), \\
& x_{1}=\varphi_{1}(t)+M_{1} \cos (n t)+N_{1} \sin (n t),
\end{aligned}
$$

and the $i$-th power solution could be expressed in the same manner.

In the general case, $M_{0}$ and $N_{0}$ could be found from the equations:

$$
\begin{aligned}
& \int_{0}^{2 \pi} F\left(t, \varphi_{0}+M_{0} \cos (n t)+N_{0} \sin (n t),\right. \\
& \left.\dot{\varphi}_{0}-M_{0} n \sin (n t)+N_{0} n \cos (n t), 0\right) \sin (n t) d t=0, \\
& \int_{0}^{2 \pi} F\left(t, \varphi_{0}+M_{0} \cos (n t)+N_{0} \sin (n t),\right. \\
& \left.\dot{\varphi}_{0}-M_{0} n \sin (n t)+N_{0} n \cos (n t), 0\right) \cos (n t) d t,
\end{aligned}
$$


where $\varphi_{0}$ is the solution of equation (1) in the nonresonant case, excluding resonant harmonics. Other coefficients $M_{i}$ and $N_{i}$ could be found from the system of linear algebraic equations:

$$
\begin{aligned}
& M_{i-1} \int_{0}^{2 \pi}\left\{\left(\frac{\partial F}{\partial x}\right) \cos (n t)-n\left(\frac{\partial F}{\partial \dot{x}}\right) \sin (n t)\right\} \cos (n t) d t \\
& +N_{i-1} \int_{0}^{2 \pi}\left\{\left(\frac{\partial F}{\partial x}\right) \sin (n t)+n\left(\frac{\partial F}{\partial \dot{x}}\right) \cos (n t)\right\} \cos (n t) d t \\
& +\int_{0}^{2 \pi} F_{i}^{*} \cos (n t) d t=0, \\
& M_{i-1} \int_{0}^{2 \pi}\left\{\left(\frac{\partial F}{\partial x}\right) \cos (n t)-n\left(\frac{\partial F}{\partial \dot{x}}\right) \sin (n t)\right\} \sin (n t) d t \\
& +N_{i-1} \int_{0}^{2 \pi}\left\{\left(\frac{\partial F}{\partial x}\right) \sin (n t)+n\left(\frac{\partial F}{\partial \dot{x}}\right) \cos (n t)\right\} \sin (n t) d t \\
& +\int_{0}^{2 \pi} F_{i}^{*} \sin (n t) d t=0,
\end{aligned}
$$

where

$$
F_{i}^{*}=F_{i}+\left(\frac{\partial F}{\partial x}\right) \varphi_{i-1}+\left(\frac{\partial F}{\partial \dot{x}}\right) \dot{\varphi}_{i-1}
$$

are known periodical functions.

The small parameter method was realized in VIBRAN for both resonant and nonresonant cases in systems of nonlinear differential equations [6].

\section{Realization of the Harmonic Balance Method}

The harmonic balance method is probably the oldest analytical method in the theory of nonlinear vibration [6]. Consider the following nonlinear differential equation:

$$
\ddot{x}+f(x, \dot{x})=F(t),
$$

where $x, \dot{x}, \ddot{x}$ denote displacement, velocity and acceletation; $f(x, \dot{x})$ is a nonlinear function, expandable in Fourier series; $F(t)$ is assumed to be a periodical function:

$$
F(t)=A_{0}+\sum_{i=1}^{\infty}\left(A_{i} \cos (i \omega t)+B_{i} \sin (i \omega t)\right) .
$$


The solution of the above mentioned equation (5) can be expressed in the Fourier series in time:

$$
x(t)=a_{0}+\sum_{i=1}^{\infty}\left(a_{i} \cos (i \omega t)+b_{i} \sin (i \omega t)\right) .
$$

A nonlinear function is also expanded in the Fourier series in time:

$$
f(x, \dot{x})=\alpha_{0}+\sum_{i=1}^{\infty}\left(\alpha_{i} \cos (i \omega t)+\beta_{i} \sin (i \omega t)\right),
$$

where Fourier series coefficients are calculated using the following formulas:

$$
\begin{aligned}
\alpha_{0} & =\frac{\omega}{2 \pi} \int_{0}^{2 \pi / \omega} f(x, \dot{x}) d t ; \quad \alpha_{i}=\frac{\omega}{\pi} \int_{0}^{2 \pi / \omega} f(x, \dot{x}) \cos (i \omega t) d t \\
\beta_{i} & =\frac{\omega}{\pi} \int_{0}^{2 \pi / \omega} f(x, \dot{x}) \sin (i \omega t) d t .
\end{aligned}
$$

A substitution of formulas (6) and (7) into equation (5) gives an infinite number of algebraic equations to determine unknown coefficients of solution (6):

$$
\begin{aligned}
& \alpha_{0}=A_{0} \text {, } \\
& i^{2} \omega^{2} a_{i}=\alpha_{i}-A_{i}, \\
& i^{2} \omega^{2} b_{i}=\beta_{i}-B_{i}, \quad i=1,2,3, \ldots, \\
& \begin{aligned}
\alpha_{0} & =\alpha_{0}\left(a_{0}, a_{1}, b_{1}, \ldots\right), \\
\text { or } \quad & \alpha_{i}=\alpha_{i}\left(a_{0}, a_{1}, b_{1}, \ldots\right), \\
& \beta_{i}=\beta_{i}\left(a_{0}, a_{1}, b_{1}, \ldots\right) .
\end{aligned}
\end{aligned}
$$

The above mentioned version of the harmonic balance method was realized in VIBRAN for the system of nonlinear equations [5].

\section{Efficiency Assessment for Both Methods}

Two VIBRAN programs that realize the above mentioned methods were tested for the following equation:

$$
\ddot{x}+c_{1} \dot{x}+c_{2} x+c_{3} x^{2}+c_{4} x^{3}=d_{0}+d_{1} \sin (t)+d_{2} \cos (t) .
$$

This equation describes dynamics of aerodynamically supported magnetic head in the recording device 8 .

We present below the result of solution for the first harmonics, the first index for solution coefficients $A$ and $B$ is the equation number and the second one is the harmonics number. First result corresponds to the first equation in formula (8) and afterwards we present the result for cosine and sine coefficients of the first term of Fourier series.

The result for the first equation of formula (8) is: 


$$
0=\mathrm{A} 10^{*} \mathrm{C} 2-.5 * \mathrm{~B} 11^{* *} 2^{*} \mathrm{C} 3-.5^{*} \mathrm{~A} 11^{* *} 2^{*} \mathrm{C} 3-\mathrm{C} 3 * \mathrm{~A} 10^{* *} 2
$$

\section{$+.75^{*} \mathrm{C} 4 * \mathrm{~B} 11^{* *} 2^{*} \mathrm{~A} 12$}

$$
\begin{aligned}
& -1.5^{*} \mathrm{C} 4^{*} \mathrm{~B} 11^{* *} 2^{*} \mathrm{~A} 10-1.5^{*} \mathrm{C} 4{ }^{*} \mathrm{~B} 11^{*} \mathrm{~B} 12^{*} \mathrm{~A} 11-1.5^{*} \mathrm{C} 4{ }^{*} \mathrm{~B} 12^{* *} 2^{*} \mathrm{~A} 10 \\
& -.75^{*} \mathrm{C} 44^{*} \mathrm{~A} 11^{* *} 2^{*} \mathrm{~A} 12-1.5^{*} \mathrm{C} 4^{*} \mathrm{~A} 11^{* *} 2^{*} \mathrm{~A} 10-1.5^{*} \mathrm{C} 4^{*} \mathrm{~A} 12^{* *} 2^{*} \mathrm{~A} 10 \\
& -\mathrm{C} 4^{*} \mathrm{~A} 10^{* *} 3-\mathrm{D} 0 .
\end{aligned}
$$

The result for the cosine coefficient of the first term of Fourier series is:

$$
\begin{aligned}
0= & -\mathrm{A} 11-\mathrm{B} 11^{*} \mathrm{C} 1+\mathrm{A} 11^{*} \mathrm{C} 3-\mathrm{B} 11^{*} \mathrm{~B} 12^{*} \mathrm{C} 3-2^{*} \mathrm{~A} 11^{*} \mathrm{~A} 10^{*} \mathrm{C} 3-\mathrm{A} 11^{*} \mathrm{~A} 12^{*} \mathrm{C} 3 \\
& -.75^{*} \mathrm{C} 4^{*} \mathrm{~B} 11^{* *} 2^{*} \mathrm{~A} 11-3^{*} \mathrm{C} 4^{*} \mathrm{~B} 11^{*} \mathrm{~B} 12^{*} \mathrm{~A} 10-1.5^{*} \mathrm{C} 4^{*} \mathrm{~B} 12^{* *} 2^{*} \mathrm{~A} 11 \\
& -.75^{*} \mathrm{C} 4^{*} \mathrm{~A} 11^{* *} 3-1.5^{*} \mathrm{C} 4^{*} \mathrm{~A} 11^{*} \mathrm{~A} 12^{* *} 2-3^{*} \mathrm{C} 4^{*} \mathrm{~A} 11^{*} \mathrm{~A} 10^{* *} 2 \\
& -3^{*} \mathrm{C} 4^{*} \mathrm{~A} 11^{*} \mathrm{~A} 12^{*} \mathrm{~A} 10-\mathrm{D} 2 .
\end{aligned}
$$

The result for the sine coefficient of the first term of Fourier series is as follows:

$$
\begin{aligned}
0= & -\mathrm{B} 11-\mathrm{A} 11^{*} \mathrm{C} 1+\mathrm{B} 11^{*} \mathrm{C} 3-\mathrm{B} 11^{*} \mathrm{~A} 12^{*} \mathrm{C} 3-2^{*} \mathrm{~B} 11^{*} \mathrm{~A} 10^{*} \mathrm{C} 3-\mathrm{A} 11^{*} \mathrm{~B} 12^{*} \mathrm{C} 3 \\
& -.75^{*} \mathrm{C} 4^{*} \mathrm{~B} 11^{*} 3+3^{*} \mathrm{C} 4^{*} \mathrm{~B} 11^{*} \mathrm{~A} 12^{*} \mathrm{~A} 10-1.5^{*} \mathrm{C} 4^{*} \mathrm{~B} 12^{* *} 2^{*} \mathrm{~B} 11 \\
& -.75^{*} \mathrm{C} 4^{*} \mathrm{~A} 11^{* *} 2^{*} \mathrm{~B} 11-1.5^{*} \mathrm{C} 4^{*} \mathrm{~B} 11^{*} \mathrm{~A} 12^{* *} 2-3^{*} \mathrm{C} 4^{*} \mathrm{~B} 11^{*} \mathrm{~A} 10^{* *} 2 \\
& -3^{*} \mathrm{C} 4^{*} \mathrm{~A} 11^{*} \mathrm{~B} 12^{*} \mathrm{~A} 10-\mathrm{D} 1 .
\end{aligned}
$$

In Figure 2, the number of terms in the solution expression (M) for the small parameter (upper column) and for the harmonic balance method (lower column) with respect to the number of harmonics (n) in the solution (number of $\mu$ power in the case of small parameter method) are presented.

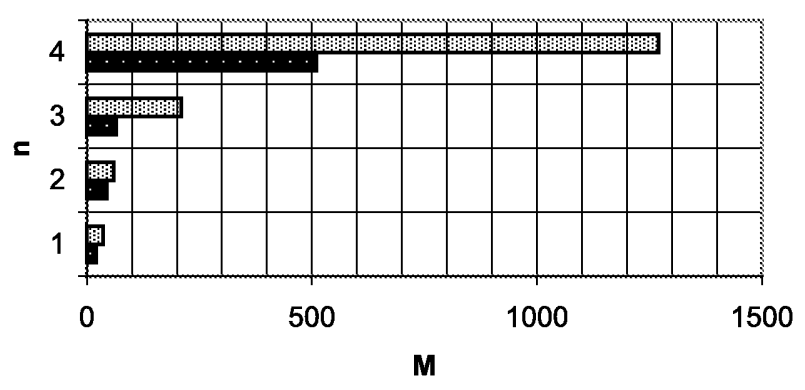

Fig. 2. Number of terms in the solution's expression.

In Figure 3, the presented graphics illustrates the convergence of above mentioned analytical methods for the four coefficients. The upper curve corresponds to the harmonic balance method and the lower one corresponds to the small parameter method.

In this case, the magnetic head construction parameters were:

$$
\begin{aligned}
& c_{1}=0.1, \quad c_{2}=0.228 \times 10^{6} \quad c_{3}=0.167 \times 10^{4}, \\
& c_{4}=0.587 \times 10^{3}, d_{0}=0, \quad d_{1}=0.12, \quad d_{2}=0.12 .
\end{aligned}
$$




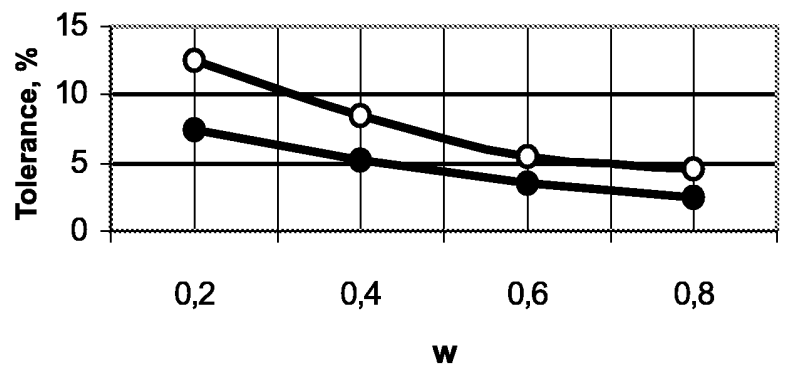

Fig. 3. Tolerance (\%) for harmonic balance and small parameter analytical methods.

\section{Conclusions}

The comparison of the abovementioned analytical methods illustrates similarities and differences of their application. The similarities illustrate tolerance curves, that are of the same shape.

There are 12045 terms in the solution's expression for the small parameter method (5 harmonics), meanwhile only 1524 terms are observed for the harmonic balance method. It means that the harmonic balance method is much more convenient for computer algebra realization, especially for the systems with multi-degree-freedom, but needs a special stability analysis procedure for the steady-state solution.

\section{References}

1. R. Lewandowski, Computational formulation for periodic vibration of geometrically nonlinear structures, Part 1: Theoretical background, Int. J. Solids Structures, 34(15), 1925-1947 (1997).

2. E. Riks, Some computational aspects of the atability analysis of nonlinear structures, Computational Methods in Applied Mechanical Engineering, 47, 219-259 (1984).

3. D.M. Klimov, V.M. Rudenko, Metody kompiuternoi algebry v zadacach mechaniki, Nauka, Moskva (1989).

4. R. Kulvietiene, G. Kulvietis, Analytical Computation Using Microcomputers, LUSTI, Vilnius (1989) (in Russian).

5. R. Kulvietiene, G. Kulvietis, J. Galkauskaite, Computer algebra application for large nonlinear oscillation systems, Mechanine technologija, 24, 126-130 (1997).

6. R. Kulvietiene, G. Kulvietis, Symbolic solution of nonlinear oscillation system using harmonic balance method, Proc. of the $2^{\text {nd }}$ European Nonlinear Oscillations Conference, Vol. 2, Prague, 109-112 (1996).

7. I.G. Malkin, Nekotorye zadaci teorii nelineinyx kolebaniy, Gostexizdat, Moskva (1956).

8. R. Kulvietiene, Dynamics of aerodynamically supported magnetic head in the recording device, Ph.D. Thesis, KTU, Kaunas, (1982).

9. F. San-Juan, A. Abad, Algebraic and symbolic manipulation of Poisson series, Journal of Symbolic Computation, 32 (5), 565-572 (2001). 